\title{
Fabrication of tunable hierarchical MXene@AuNPs nanocomposites constructed by self-reduction reactions with enhanced catalytic performances
}

\author{
Kaikai Li $^{1,2}$, Tifeng Jiao ${ }^{1,2^{*}}$, Ruirui Xing ${ }^{2,3}$, Guodong Zou ${ }^{1}$, Jingxin Zhou ${ }^{2}$, Lexin Zhang ${ }^{2}$ and \\ Qiuming Peng ${ }^{1^{*}}$
}

\begin{abstract}
MXene, a new type of two-dimensional layered transition metal carbide material differing from graphene, demonstrates intriguing chemical/physical properties and wide applications in recent years. Here, the preparation of the self-assembled MXene-gold nanoparticles (MXene@AuNPs) nanocomposites with tunable sizes is reported. The nanocomposites are obtained via the self-reduction reactions of MXene material in a $\mathrm{HAuCl}_{4}$ solution at room temperature. The sizes of the Au particles can be well-controlled by regulating the self-reduction reaction time. They can greatly influence the catalytic behaviors of the MXene@AuNPs composites. MXene@AuNPs composites with optimized reduction time show high catalytic performances and good cycle stability for model catalytic reactions of nitro-compounds, such as 2-nitrophenol and 4-nitrophenol. This work demonstrates a new approach for the preparation of tunable MXenebased self-assembled composites.
\end{abstract}

Keywords: MXene, self-reduction, gold nanoparticle, nitrocompound, nanocomposites

\section{INTRODUCTION}

Various kinds of two-dimensional (2D) materials have drawn increasing attention in recent years, owing to special physical and chemical properties [1-9]. In 2011, Naguib first prepared transition metal nitride/carbon nano-layered materials (MXene), a new type of 2D layer material [10]. The earliest discovered transition metal 2D carbide/nitride is a member of the MXene family $[11,12]$. At present, MXene [13] is mainly synthesized by the corrosion of the A-group layered $\mathrm{M}_{n+1} \mathrm{AX}_{n}$ (MAX) phase by hydrofluoric acid, where $n=1$ to $3, \mathrm{M}$ is an early transition metal, A is an A-group (mostly IIIA and IVA, or groups 13 and 14) element and $\mathrm{X}$ is either carbon and/ or nitrogen [14-17]. The surface of MXene contains many active groups, such as hydroxyl groups, oxygen ions, and fluorine ions [18-20]. The 2D MXene materials show special nanostructures that easily induce oxidization reaction in environments with oxygen and water [21-24], and demonstrate special electronic [25], magnetic [26] and mechanical properties [27]. Additionally, MXene has a wide range of applications, such as for energy storage, catalysis, adsorption, hydrogen storage, sensors, and new polymer-reinforced composites. Presently, research on MXene is mainly focused on lithium ion batteries [28,29], supercapacitors and fuel cells [30-32]. Additionally, some special aspects regarding the catalytic performances of MXene materials, such as high-performance oxygen evolution, efficient electrocatalyst for hydrogen evolution and single-atom catalyst for $\mathrm{CO}$ oxidation, have been investigated [33-37]. Moreover, in recent years, our group has prepared some novel MXene/Ag composites, MXene-based nanoflower-shaped $\mathrm{TiO}_{2} / \mathrm{C}$ composites, and MXene/magnetic iron oxide nanocomposites, which demonstrate unusual electrocatalytic activity, extraordinary long cycle lifetime lithium storage and catalytic activity for the dehydrogenation of sodium alanates, respectively [38-41]. However, it is still challenging to design and obtain MXene-based composites via facile and effective self-assembly. Alternatively, Au nanostructures and nanocomposites have shown important applications in cancer-related detection, selective photocatalysts and controlled drug release [42-45]. It is expected that a combination of MXene-based composites with gold na-

\footnotetext{
${ }^{1}$ State Key Laboratory of Metastable Materials Science and Technology, Yanshan University, Qinhuangdao 066004, China

${ }^{2}$ Hebei Key Laboratory of Applied Chemistry, School of Environmental and Chemical Engineering, Yanshan University, Qinhuangdao 066004, China

${ }^{3}$ State Key Laboratory of Biochemical Engineering, Institute of Process Engineering, Chinese Academy of Sciences, Beijing 100190, China

* Corresponding authors (emails: tfjiao@ysu.edu.cn (Jiao T); pengqiuming@ysu.edu.cn (Peng Q))
} 
noparticles could be especially advantageous, owing to controllable nanostructures and enhanced catalytic properties.

In this work, we report the synthesis and characterization of new MXene-gold nanoparticles (MXene@AuNPs) composites with layered MXene $\left(\mathrm{Ti}_{3} \mathrm{C}_{2}-\right.$ $\left.\left(\mathrm{OH}_{x} \mathrm{~F}_{1-x}\right)_{2}\right)$ as a template for the direct reduction of $\mathrm{Au}$ nanoparticles in $\mathrm{HAuCl}_{4}$ solution. The layered MXene acts as a supporter and reductant in the reaction process. The obtained MXene@AuNPs composites exhibit exceptional performances for the model reduction reaction of nitro-compounds, such as 2-nitrophenol (2-NA) and 4nitrophenol (4-NP) [46-48]. Additionally, we study the size of surface-reduced gold particles and their catalytic effects on 2-NA and 4-NP at different reaction times. This present work suggests that MXene-based composites may be promising catalyst candidates, demonstrating great potential in the fields of composite catalyst materials and wastewater treatment.

\section{EXPERIMENTAL SECTION}

\section{Materials}

2-NA and 4-NP were obtained from Aladdin Reagent (Shanghai, China). Ultra-pure water was obtained through a Milli-Q Millipore filter system (Millipore Co., Bedford, MA, USA) with a resistivity of $18.2 \mathrm{M} \Omega \mathrm{cm}^{-1}$. Chloroauric acid tetrahydrate $\left(\mathrm{HAuCl}_{4} \cdot 4 \mathrm{H}_{2} \mathrm{O}\right)$ and sodium borohydride $\left(\mathrm{NaBH}_{4}\right)$ were obtained from Alfa Aesar (Beijing, China). Ethanol $\left(\mathrm{C}_{2} \mathrm{H}_{5} \mathrm{OH}\right)$ was provided by Sinopharm Chemical Reagent Co., Ltd. (Beijing, China). All chemicals were used, as received, without further purification. MXene $\left(\mathrm{Ti}_{3} \mathrm{C}_{2}\left(\mathrm{OH}_{x} \mathrm{~F}_{1-x}\right)_{2}\right)$ was synthesized according to previous reports $[39,40]$.

\section{Preparation of composites MXene@AuNPs}

First, $100 \mathrm{mg}$ of MXene solid powder, after sonication for $30 \mathrm{~min}$ in an ultrasonic water bath, was dispersed in $100 \mathrm{~mL}$ ultrapure water to obtain a uniform suspension. Then, $3 \mathrm{~mL}$ of $\mathrm{HAuCl}_{4}\left(0.1 \mathrm{~mol} \mathrm{~L}^{-1}\right)$ was slowly added to the suspension and constantly stirred to give full response. After reacting for $20 \mathrm{~min}$, the obtained composite (named as MXene@AuNPs20) suspension was centrifuged several times with ultrapure water. Finally, the composite was freeze-dried in a dryer at $-60^{\circ} \mathrm{C}$ for $48 \mathrm{~h}$. Additionally, via catalytic reduction reactions for 5 or 60 min, MXene@AuNPs5 or MXene@AuNPs60 composites, respectively, were synthesized under the same conditions.

\section{Catalytic performance of MXene@AuNPs composites}

The catalytic performance of MXene is evaluated mainly through the degradation of nitro-aromatics, including 4$\mathrm{NP}$ and 2-NA by $\mathrm{NaBH}_{4}$ [49]. The prepared MXene@AuNPs suspension $\left(0.3 \mathrm{~mL}, 1 \mathrm{mg} \mathrm{mL}^{-1}\right)$ was mixed with an aqueous solution of $2-\mathrm{NA}\left(2 \mathrm{~mL}, 5 \mathrm{mmol} \mathrm{L}^{-1}\right)$ or 4-NP $\left(2 \mathrm{~mL}, 5 \mathrm{mmol} \mathrm{L}^{-1}\right)$, and a fresh aqueous $\mathrm{NaBH}_{4}$ solution $\left(20 \mathrm{~mL}, 0.01 \mathrm{~mol} \mathrm{~L}^{-1}\right)$. The filtered solution samples were analyzed by UV-vis spectroscopy until the mixture became colorless. Additionally, we also studied the cyclic stability under the same environmental conditions.

\section{Characterization}

The morphology of the sample was characterized by fieldemission scanning electron microscopy (SEM, Hitachi S4800, Japan) and transmission electron microscopy (TEM, HT7700, Hitachi High-Technologies Corporation, Ibaraki, Japan). High resolution transmission electron microscopy (HRTEM) images were acquired with a JEM2010 electron microscope operated at $200 \mathrm{kV}$. X-ray diffraction (XRD) patterns of the samples were obtained with an X-ray diffractometer equipped with a Bragg diffraction setup (SMART LAB, Rigaku, Akishima, Japan) and a $\mathrm{Cu} \mathrm{Ka} \mathrm{X}$-ray radiation source. At different times, the reaction solution concentration was measured by ultraviolet absorption spectroscopy using a UV-2550 spectrophotometer. X-ray photoelectron spectroscopy (XPS) analysis was performed on a Thermo Scientific ESCALab 250Xi XPS (Thermo Fisher Scientific, San Jose, CA, USA) using $200 \mathrm{~W}$ monochromated $\mathrm{Al} \mathrm{Ka}$ radiation. Thermogravimetry (TG) analysis was carried out using a NETZSCH STA 409 PC Luxx simultaneous thermal analyzer (Netzsch Instruments Manufacturing Co., Ltd., Seligenstadt, Germany) in an argon atmosphere.

\section{RESULTS AND DISCUSSION}

\section{Structural characterization of MXene@AuNPs composites} The flowchart in Fig. 1 shows that this experiment mainly involves the preparation of composite MXene@AuNPs and analysis of the catalytic properties of composite MXene@AuNPs on nitro-compounds. First, under ambient conditions, MXene reacted with $\mathrm{HAuCl}_{4}$ to obtain composite MXene@AuNPs with different particle sizes of Au. Next, the catalytic properties of two nitro-compounds 2-NA and 4-NP were studied with the composite MXene@AuNPs.

The SEM image (Fig. 2a) shows that the original MXene is a layered structure and is more visible [50]. 


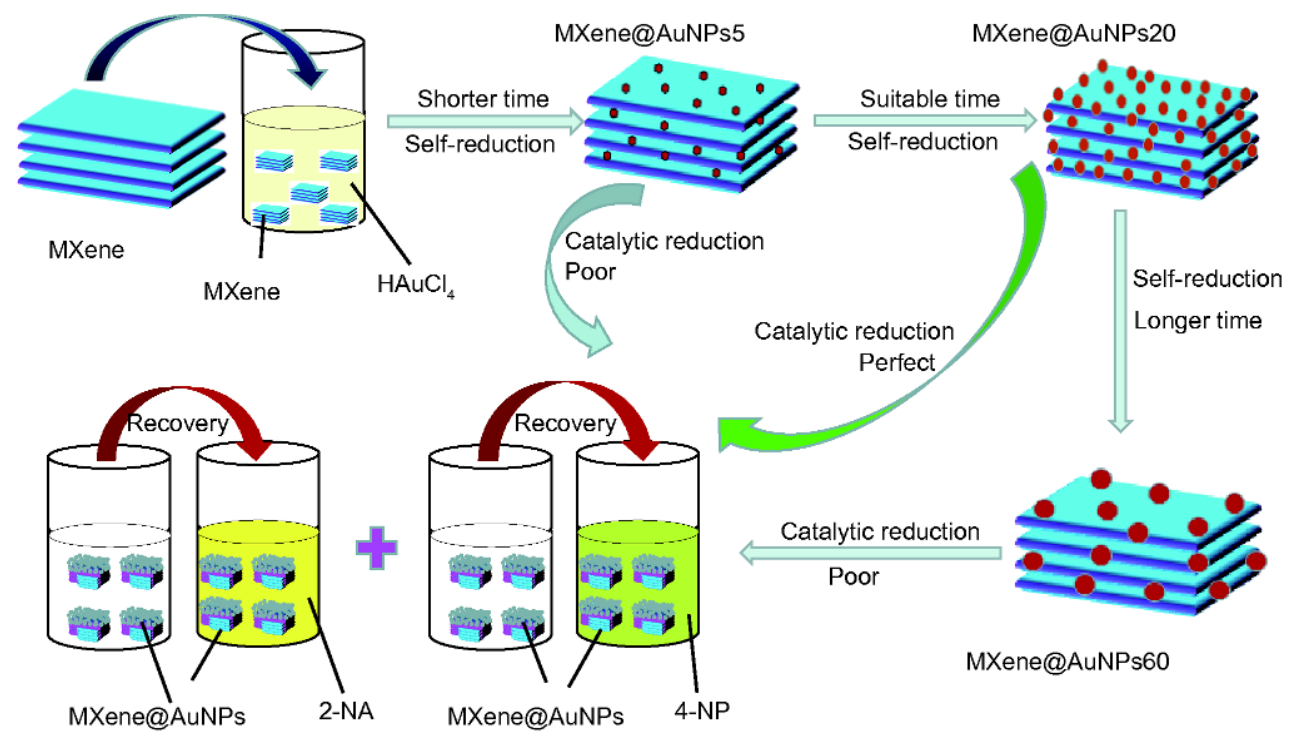

Figure 1 Schematic illustration of the fabrication and catalysis of MXene@AuNPs composites.
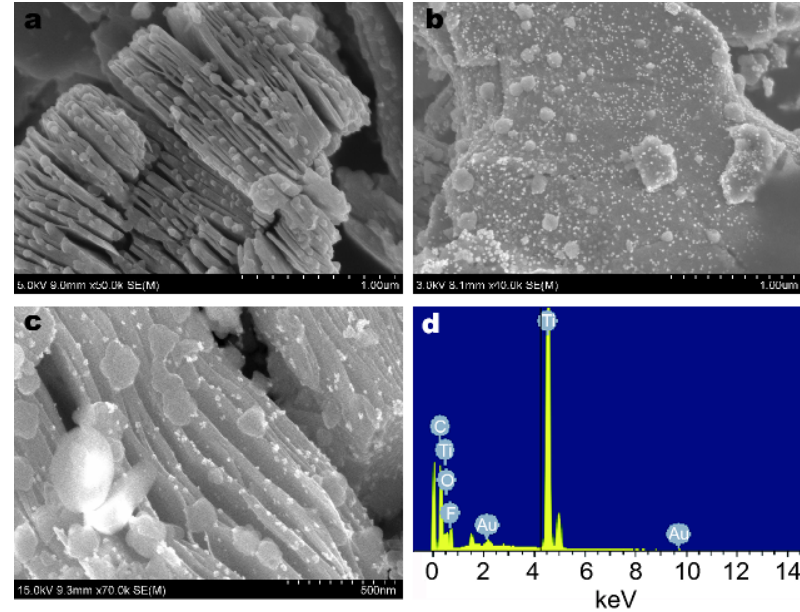

Figure 2 (a) Representative SEM image of the layered MXene sample. (b) Representative SEM image of the layered MXene@AuNPs sample. (c) High magnification image of a local area in (b). (d) Representative EDS image of the layered MXene@AuNPs sample.

After $\mathrm{HAuCl}_{4}$ treatment, the surface of MXene has many white spots, as shown in Fig. 2b, c. By energy dispersive spectrometer (EDS) analysis, it is further verified that the white spots on the MXene surface are gold particles, indicating that many gold particles are mainly distributed on the surface of MXene after $\mathrm{HAuCl}_{4}$ treatment, as shown in Figs 2d and 3. HRTEM images indicate the nanoparticles are single crystal. In the process of Au reduction by MXene, with the increase of reaction time, $\mathrm{Au}$ particles grow slowly. Lattice figures with an interplanar spacing of $d_{111}=0.238 \pm 0.07 \mathrm{~nm}$ and $d_{200}=0.210 \pm 0.01 \mathrm{~nm}$ are clearly visible, as shown in Fig. $3 \mathrm{~b}$, e, h. The average particle size of the $\mathrm{Au}$ particles is concentrated at $15 \pm 5 \mathrm{~nm}$ at $5 \mathrm{~min}$, as shown in Fig. 3c. Additionally, the average size of gold particles in MXene@AuNPs20 and MXene@AuNPs60 composites are 25 \pm 5 nm (Fig. 3f) and $35 \pm 5 \mathrm{~nm}$ (Fig. 3i), respectively, indicating that the particle size of gold particles increases slowly with the increment of reaction time.

The XRD pattern (Fig. 4) shows four major diffraction peaks, which can be attributed to the (111), (200), (220), and (311) planes of face-centered-cubic Au single crystal. With the increment of reaction time, the peaks of $\mathrm{Au}$ become sharper and stronger, indicating the enhancement of Au crystallinity. Additionally, the (002) peak intensity of MXene at $\sim 8.9^{\circ}$ becomes weak, which could be speculated to be the partial oxidation of the MXene sheet in the reduction reaction process of gold nanoparticles [51].

As shown in Fig. 5, the thermal stability of MXene and MXene@AuNPs composite was evaluated by TG, which is an important technique to investigate functional nanocomposite materials [52-56]. TG results show the final heat loss at $650^{\circ} \mathrm{C}$, in which the maximum mass loss of MXene@AuNPs60 is about $12.3 \%$, followed by MXene@AuNPs20, MXene@AuNPs5, and MXene. With the increment of reaction time, the heat loss of composite MXene@AuNPs is also larger, indicating that the thermal stability of the composite MXene@AuNPs60 worsens.

XPS is a useful tool to investigate the chemical states 

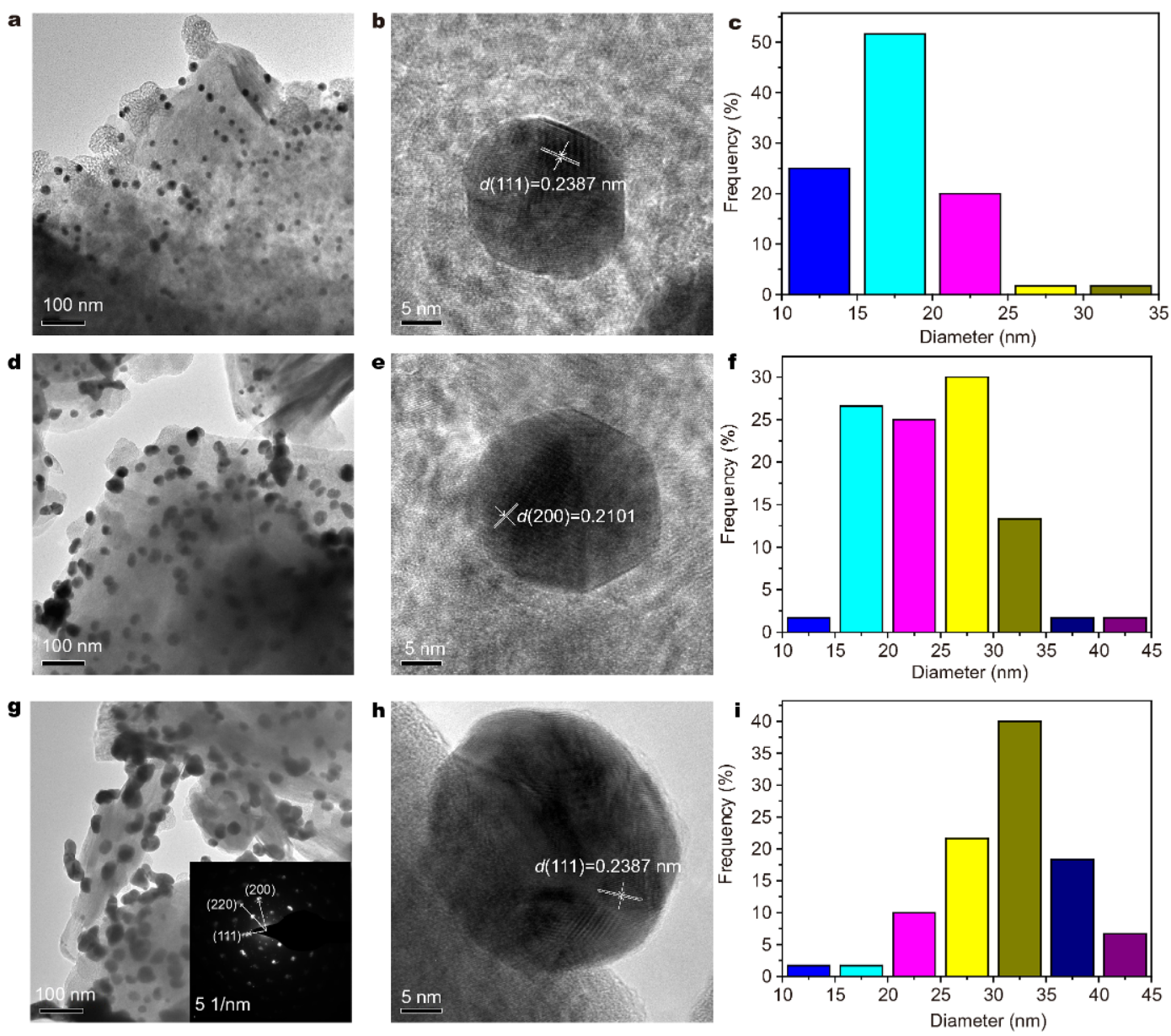

Figure 3 (a, d, g) TEM images of the prepared MXene@AuNPs5, MXene@AuNPs20, and MXene@AuNPs60 samples at 5, 20, and 60 min. (b, e, h) High-resolution TEM images of Au nanoparticles at (a, d, g). (c, f, i) Particle size distribution of Au nanoparticles.

and elemental components in various functional composites [57-61]. To study the mechanism of self-reduction, XPS analysis was performed for the MXene and MXene@AuNPs60 composite (Fig. 6a). The obvious peak at $\sim 458.8 \mathrm{eV}$ from original MXene samples is assigned to Ti(IV) $2 p_{3 / 2}$ (Fig. 6b). Simultaneously, the broad peak ranging from $454-457 \mathrm{eV}$ in Fig. $6 \mathrm{~b}$ suggests the possible presence of low valence $\mathrm{Ti}$ species (i.e., $\mathrm{Ti}(\mathrm{II})(\sim 454.7 \mathrm{eV})$ and $\operatorname{Ti}(\mathrm{III})(\sim 455.6 \mathrm{eV}))[62,63]$. On the other hand, the self-reduction process onto a MXene@AuNPs60 composite can lead to the initial conversion from $\mathrm{Ti}$ (II) and $\mathrm{Ti}$ (III) species to the termination of the $\operatorname{Ti}(\mathrm{IV})$ species, as shown in Fig.6c. The MXene@AuNPs60 composite (Fig. 6d) shows peaks at $82.3 \mathrm{eV}$ and $86.1 \mathrm{eV}$, where the binding energy difference is $3.6 \mathrm{eV}$, indicating a reduced form of $\mathrm{Au}(0)$, thus further confirming the presence of $\mathrm{Au}$ nanoparticles in the sample. The obtained results indicate that all gold elements exist in the $\mathrm{Au}(0)$ (i.e., metallic) state, which further proves the strong reductive activity in low valence $\mathrm{Ti}(\mathrm{II})$ and $\mathrm{Ti}(\mathrm{III})$ species. Therefore, the self-reduction of $\mathrm{Au}$ is ascribed to the activated low valence Ti species.

\section{Catalytic performances of MXene@AuNPs composites}

The catalytic capability of the obtained MXene@AuNPs nanocomposites was evaluated by the catalytic hydrogenation reaction of nitro-compounds [64]. In this study, the reduction of 2 -NA $\left(2 \mathrm{~mL}, 5 \mathrm{mmol} \mathrm{L}^{-1}\right)$ or $4-\mathrm{NP}$ $\left(2 \mathrm{~mL}, 5 \mathrm{mmol} \mathrm{L}^{-1}\right)$ with fresh aqueous $\mathrm{NaBH}_{4}(20 \mathrm{~mL}$, $\left.0.01 \mathrm{~mol} \mathrm{~L}^{-1}\right)$ at room temperature was performed to investigate the catalytic activity of the composite MXene@AuNPs20, as monitored by UV-vis spectroscopy. After the addition of $\mathrm{NaBH}_{4}$ solution to the 4-NP solution, the UV-Vis absorption peak of 4-NP was from 317 to $402 \mathrm{~nm}$, indicating the formation of 4-nitrophenolate [65]. In the absence of a catalyst, the color of the mixture 


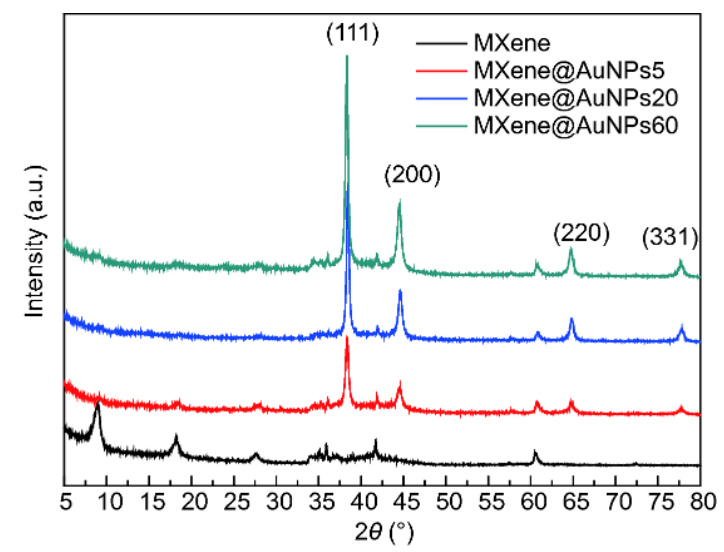

Figure 4 XRD patterns of the prepared different MXene composites.
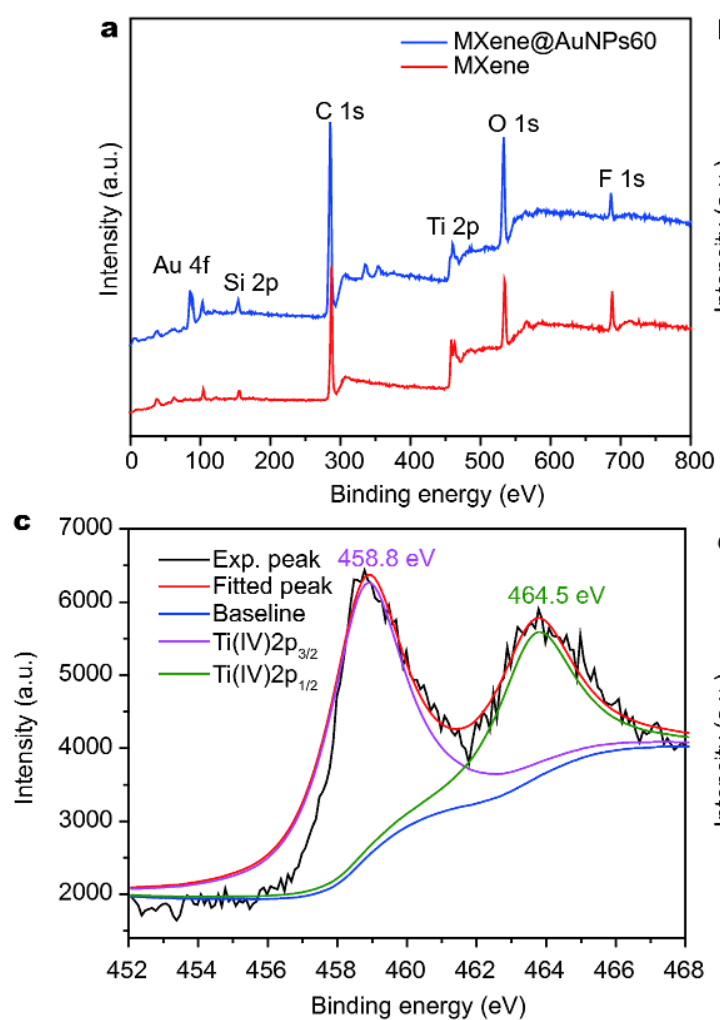

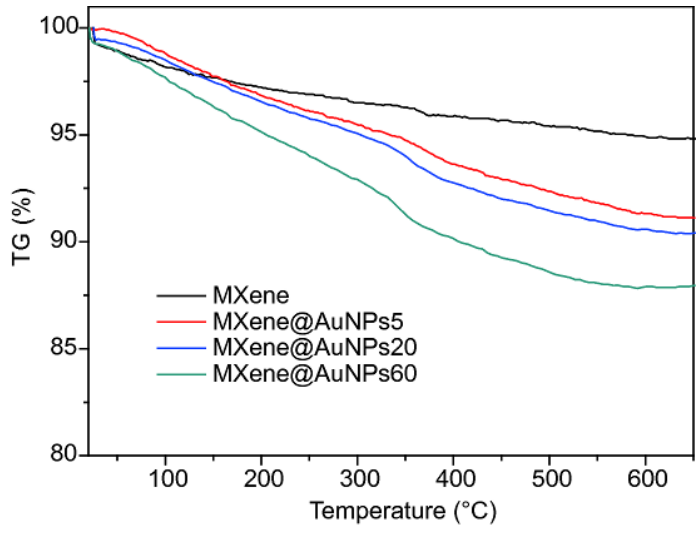

Figure 5 TG curves of MXene, MXene@AuNPs5, MXene@AuNPs20, and MXene@AuNPs60.
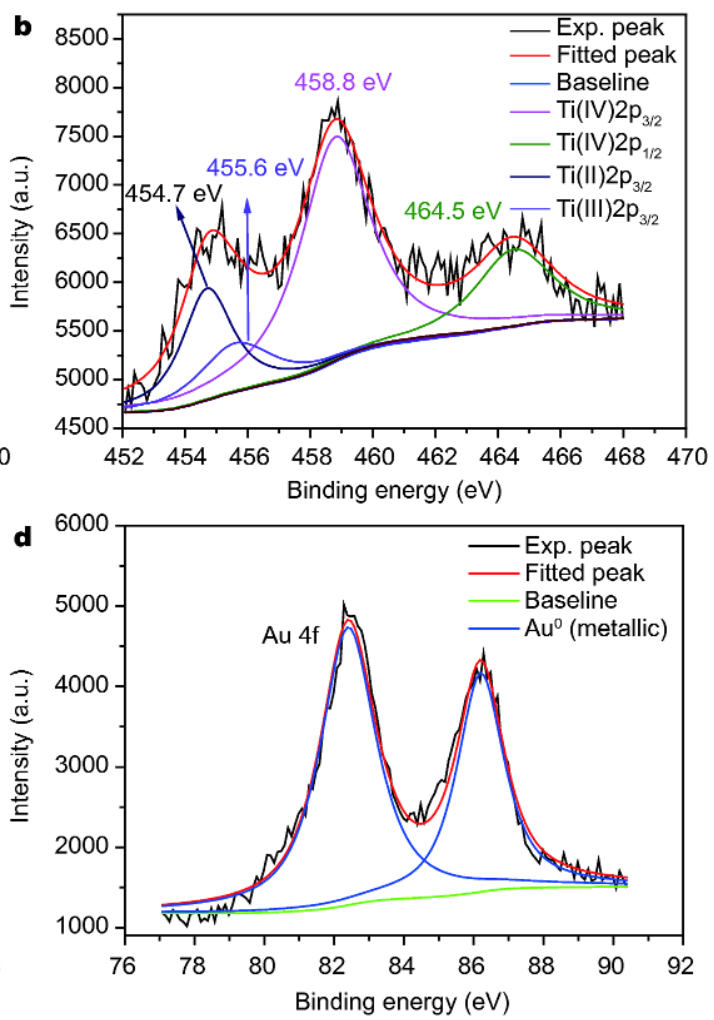

Figure 6 (a) XPS profiles of the MXene and MXene@AuNPs60 samples; (b) Ti 2p in the MXene; (c) Ti 2p in the MXene@AuNPs60; (d) Au 4f in the MXene@AuNPs60.

of 4-NP and $\mathrm{NaBH}_{4}$ does not change significantly for $24 \mathrm{~h}$, indicating that the reaction does not occur. Upon addition of a small amount of composite MXene@AuNPs20 suspension, the mixture gradually became colorless over time. The typical peak of $4-\mathrm{NP}$ at $402 \mathrm{~nm}$ gradually diminished, indicating the complete reduction of 4-NP (Fig. 7a). Considering that the concentration of $\mathrm{NaBH}_{4}$ is much higher than that of $4-\mathrm{NP}\left(C_{\mathrm{NaBH} 4} / C_{4-\mathrm{NP}}=\right.$
400), the whole reaction process of 4-NP can be regarded as a pseudo first-order reaction. A linear relationships between $\ln \left(C_{t} / C_{0}\right)$ and time $(t)$ of the MXene@AuNPs20 catalyst (Fig. 7b) indicates that the catalytic reaction of 4NP could be well-considered as a pseudo-first-order reaction, where $C_{t}$ is the concentration at time $t$, and $C_{0}$ represents the initial concentration. The rate of the pseudo-first-order reaction is $0.175 \mathrm{~min}^{-1}$, indicating that 


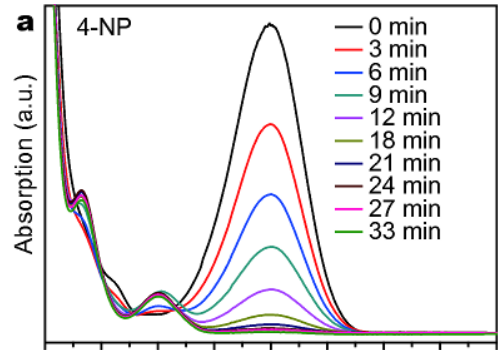

200250300350400450500550600 Wavelength $(\mathrm{nm})$

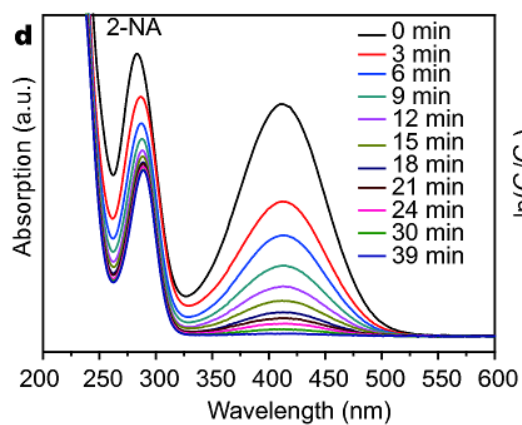

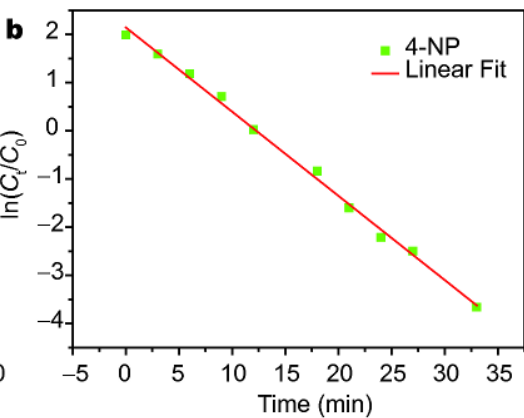
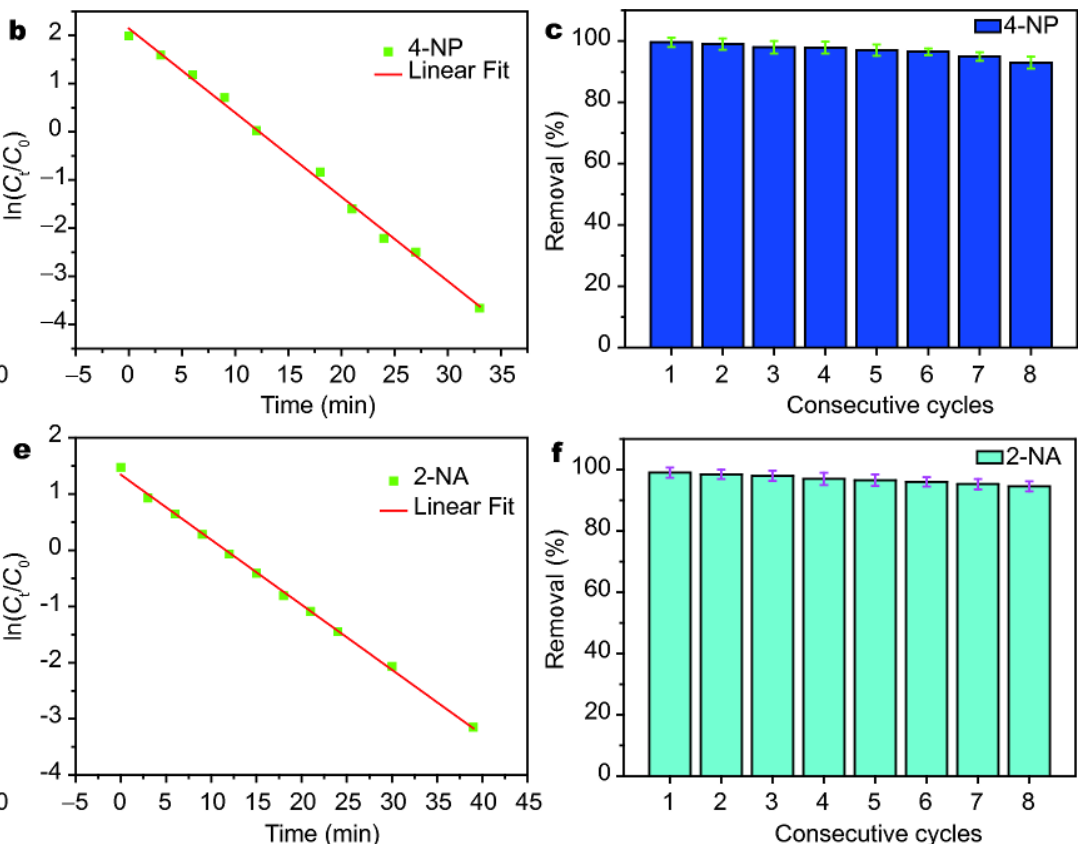

Figure 7 (a, d) Catalytic reduction of 4-NP or 2-NA with MXene@AuNPs20 composite. (b, e) The relationship between ln $\left(C_{t} / C_{0}\right)$ and the reaction time $(t)$ of the nanocomposite catalyst. (c, f) The recyclable catalysis capacities of MXene@AuNPs20 composite for the reduction reaction of 4-NP or 2-NA by $\mathrm{NaBH}_{4}$.

the composite MXene@AuNPs20 had excellent catalytic activity for 4-NP. After eight consecutive catalytic cycles, the catalytic conversion was still more than 92\%, indicating its superior stability in comparison with other kinds of composite catalysts [66-71], as shown in Fig. 7c. Additionally, we have studied the catalytic mixtures of MXene@AuNPs5 and MXene@AuNPs60 composites, and linear-fitted their data (Fig. S1). As shown in Fig. S1a, b, the MXene@AuNPs5 composite can completely catalyze 4-NP mixtures within $1 \mathrm{~h}$ with a well linear dependence. The MXene@AuNPs60 composite fully catalyzes the 4-NP mixture slightly earlier than the MXene@AuNPs5 composite, but slightly later than the MXene@AuNPs20 composite, and the reaction order is close to 1 , indicating that the MXene@AuNPs20 composite is an ideal catalyst (Fig. S1c, d).

Catalytic reduction of 2-NA was also carried out to evaluate the activity of gold nanoparticles [72]. In the absence of a catalyst, the color of the $\mathrm{NaBH}_{4}$ solution and 2-NA mixture did not change significantly over $24 \mathrm{~h}$, and the intensity change of the characteristic absorption band of solution at $415 \mathrm{~nm}$ was negligible. After adding a small amount of the composite MXene@AuNPs20, the mixed solution turned colorless within $1 \mathrm{~h}$, indicating that the MXene@AuNPs20 composite can also be almost completely catalyze the 2-NA mixture solution (Fig. 7d). Like the MXene@AuNPs20 composite-catalyzed reduction of 4-NP, the MXene@AuNPs20 composite-catalyzed reduction of 2-NA can be also considered as a pseudo-firstorder reaction (Fig. 7e). The rate of the pseudo-first-order reaction is $0.116 \mathrm{~min}^{-1}$, indicating that the composite MXene@AuNPs20 has excellent catalytic activity for 2NA. The rate of the pseudo-first-order reaction for 4-NP is much higher than that of 2-NA, indicating that the catalytic effect of 4-NP is better than that of 2-NA. After eight catalytic cycles, the conversion rate of the catalyst remained above 95\% (Fig. 7f). The MXene@AuNPs5 and MXene@AuNPs60 composites can catalyze the 2-NA mixture completely within a certain period; however, the time required is slightly higher than that of the composite MXene@AuNPs20 (Fig. S2), indicating that the MXene@AuNPs20 composite is a highly efficient catalyst for the catalytic reaction of 2-NA. Additionally, the catalytic effect of pure gold particles on 4-NP or 2-NA is far less than that of MXene@AuNPs composites, indicating that the MXene@AuNPs composite is an excellent catalyst.

\section{CONCLUSIONS}

In summary, new MXene@AuNPs nanocomposites were prepared via a self-reduction reaction of MXene with $\mathrm{HAuCl}_{4}$. The catalytic performances of the composite MXene@AuNPs were obviously affected by the time of 
the self-reduction reaction. By controlling the duration of the self-reduction reaction, MXene@AuNPs composites with different particle sizes of $\mathrm{Au}$ particles were prepared. Owing to the special structural features and excellent $\mathrm{Au}$ based catalytic activity, the composite MXene@AuNPs exhibited excellent reduction ability for some nitro compounds, such as 2-NA and 4-NP. Experimental results show that the composite MXene@AuNPs20 prepared with suitable self-reduction reaction time has the best catalytic effect. Additionally, even after eight successive catalytic cycles, the composite MXene@AuNPs showed great stability. Thus, this work presents a new route for the preparation of MXene-based nanocomposite materials.

Received 21 November 2017; accepted 25 December 2017; published online 17 January 2018

1 Ghidiu M, Lukatskaya MR, Zhao MQ, et al. Conductive two-dimensional titanium carbide 'clay' with high volumetric capacitance. Nature, 2014, 3: 78-81

2 Jian M, Wang C, Wang Q, et al. Advanced carbon materials for flexible and wearable sensors. Sci China Mater, 2017, 60: 10261062

$3 \mathrm{Li} \mathrm{Y}$. Hybrid atomic layers based electrocatalyst converts waste $\mathrm{CO}_{2}$ into liquid fuel. Sci China Mater, 2016, 59: 1-3

$4 \mathrm{Li} \mathrm{HH}, \mathrm{Yu} \mathrm{SH}$. A reviving templating method for multiple nanostructures. Sci China Mater, 2017, 60: 461-462

5 Duan X. Hot graphene sponge cleans viscous crude-oil spill. Sci China Mater, 2017, 60: 681-682

6 Ganatra R, Zhang Q. Few-layer $\mathrm{MoS}_{2}$ : a promising layered semiconductor. ACS Nano, 2014, 8: 4074-4099

7 Lukatskaya MR, Dunn B, Gogotsi Y. Multidimensional materials and device architectures for future hybrid energy storage. Nat Commun, 2016, 7: 12647

8 Wang $\mathrm{X}$, Cai A, Wen $\mathrm{X}$, et al. Graphene oxide- $\mathrm{Fe}_{3} \mathrm{O}_{4}$ nanocomposites as high-performance antifungal agents against Plasmopara viticola. Sci China Mater, 2017, 60: 258-268

9 Ge J, Lan M, Liu W, et al. Graphene quantum dots as efficient, metal-free, visible-light-active photocatalysts. Sci China Mater, 2017, 59: 12-19

10 Naguib M, Mochalin VN, Barsoum MW, et al. 25th anniversary article: MXenes: a new family of two-dimensional materials. Adv Mater, 2013, 26: 992-1005

11 Gogotsi Y. Chemical vapour deposition: transition metal carbides go 2D. Nat Mater, 2015, 14: 1079-1080

12 Naguib M, Kurtoglu M, Presser V, et al. Two-dimensional nanocrystals produced by exfoliation of $\mathrm{Ti}_{3} \mathrm{AlC}_{2}$. Adv Mater, 2011, 23: 4248-4253

13 Naguib M, Mashtalir O, Carle J, et al. Two-dimensional transition metal carbides. ACS Nano, 2012, 6: 1322-1331

14 Song J, Xing R, Jiao T, et al. Crystalline dipeptide nanobelts based on solid-solid phase transformation self-assembly and their polarization imaging of cells. ACS Appl Mater Interfaces, 2018, DOI: 10.1021/acsami.7b17933

15 Mashtalir O, Naguib M, Dyatkin B, et al. Kinetics of aluminum extraction from $\mathrm{Ti}_{3} \mathrm{AlC}_{2}$ in hydrofluoric acid. Mater Chem Phys,
2013, 139: 147-152

16 Naguib M, Halim J, Lu J, et al. New two-dimensional niobium and vanadium carbides as promising materials for Li-ion batteries. J Am Chem Soc, 2013, 135: 15966-15969

17 Ying Y, Liu Y, Wang X, et al. Two-dimensional titanium carbide for efficiently reductive removal of highly toxic chromium(VI) from water. ACS Appl Mater Interfaces, 2015, 7: 1795-1803

18 Srimuk P, Kaasik F, Krüner B, et al. MXene as a novel intercalation-type pseudocapacitive cathode and anode for capacitive deionization. J Mater Chem A, 2016, 4: 18265-18271

19 Liang X, Garsuch A, Nazar LF. Sulfur cathodes based on conductive MXene nanosheets for high-performance lithium-sulfur batteries. Angew Chem Int Ed, 2015, 54: 3907-3911

20 Lu X, Tao L, Song D, et al. Bimetallic Pd@Au nanorods based ultrasensitive acetylcholinesterase biosensor for determination of organophosphate pesticides. Sensors Actuators B-Chem, 2018, 255: 2575-2581

21 Anasori B, Lukatskaya MR, Gogotsi Y. 2D metal carbides and nitrides (MXenes) for energy storage. Nat Rev Mater, 2017, 2: 16098

22 Li Z, Wang L, Sun D, et al. Synthesis and thermal stability of twodimensional carbide MXene $\mathrm{Ti}_{3} \mathrm{C}_{2}$. Mater Sci Eng-B, 2015, 191: 3340

23 Li J, Du Y, Huo C, et al. Thermal stability of two-dimensional $\mathrm{Ti}_{2} \mathrm{C}$ nanosheets. Ceramics Int, 2015, 41: 2631-2635

24 Wang K, Zhou Y, Xu W, et al. Fabrication and thermal stability of two-dimensional carbide $\mathrm{Ti}_{3} \mathrm{C}_{2}$ nanosheets. Ceramics Int, 2016, 42: 8419-8424

25 Khazaei M, Ranjbar A, Ghorbani-Asl M, et al. Nearly free electron states in MXenes. Phys Rev B, 2016, 93: 205125-205135

26 Lane NJ, Barsoum MW, Rondinelli JM. Electronic structure and magnetism in two-dimensional hexagonal $5 \mathrm{~d}$ transition metal carbides, Tan+1Cn $(n=1,2,3)$. Europhys Lett, 2013, 101: 1-5

27 Kurtoglu M, Naguib M, Gogotsi Y, et al. First principles study of two-dimensional early transition metal carbides. MRC, 2012, 2: 133-137

28 Naguib M, Come J, Dyatkin B, et al. MXene: a promising transition metal carbide anode for lithium-ion batteries. Electrochem Commun, 2012, 16: 61-64

29 Kim SJ, Naguib M, Zhao M, et al. High mass loading, binder-free MXene anodes for high areal capacity Li-ion batteries. Electrochim Acta, 2015, 163: 246-251

30 Halim J, Lukatskaya MR, Cook KM, et al. Transparent conductive two-dimensional titanium carbide epitaxial thin films. Chem Mater, 2014, 26: 2374-2381

31 Lukatskaya MR, Mashtalir O, Ren CE, et al. Cation intercalation and high volumetric capacitance of two-dimensional titanium carbide. Science, 2013, 341: 1502-1505

32 Wang $\mathrm{X}$, Kajiyama S, Iinuma $\mathrm{H}$, et al. Pseudocapacitance of MXene nanosheets for high-power sodium-ion hybrid capacitors. Nat Commun, 2015, 6: 6544

33 Ma TY, Cao JL, Jaroniec M, et al. Interacting carbon nitride and titanium carbide nanosheets for high-performance oxygen evolution. Angew Chem Int Ed, 2016, 55: 1138-1142

34 Seh ZW, Fredrickson KD, Anasori B, et al. Two-dimensional molybdenum carbide (MXene) as an efficient electrocatalyst for hydrogen evolution. ACS Energ Lett, 2016, 1: 589-594

35 Zhang $\mathrm{X}$, Lei J, Wu D, et al. A Ti-anchored $\mathrm{Ti}_{2} \mathrm{CO}_{2}$ monolayer (MXene) as a single-atom catalyst for $\mathrm{CO}$ oxidation. J Mater Chem A, 2016, 4: 4871-4876 
Ran J, Gao G, Li FT, et al. $\mathrm{Ti}_{3} \mathrm{C}_{2}$ MXene co-catalyst on metal sulfide photo-absorbers for enhanced visible-light photocatalytic hydrogen production. Nat Commun, 2017, 8: 13907

37 Guo Z, Zhou J, Zhu L, et al. MXene: a promising photocatalyst for water splitting. J Mater Chem A, 2016, 4: 11446-11452

38 Zhang $\mathrm{Z}, \mathrm{Li} \mathrm{H}$, Zou G, et al. Self-reduction synthesis of new MXene/Ag composites with unexpected electrocatalytic activity. ACS Sustain Chem Eng, 2016, 4: 6763-6771

39 Zou G, Zhang Z, Guo J, et al. Synthesis of MXene/Ag composites for extraordinary long cycle lifetime lithium storage at high rates. ACS Appl Mater Interfaces, 2016, 8: 22280-22286

40 Zou G, Liu B, Guo J, et al. Synthesis of nanoflower-shaped MXene derivative with unexpected catalytic activity for dehydrogenation of sodium alanates. ACS Appl Mater Interfaces, 2017, 9: 7611-7618

41 Zhang Q, Teng J, Zou G, et al. Efficient phosphate sequestration for water purification by unique sandwich-like MXene/magnetic iron oxide nanocomposites. Nanoscale, 2016, 8: 7085-7093

42 Yang Y, Jiang X, Chao J, et al. Synthesis of magnetic core-branched $\mathrm{Au}$ shell nanostructures and their application in cancer-related miRNA detection via SERS. Sci China Mater, 2017, 60: 1129-1144

43 Liu W, Liu Z, Wang G, et al. Carbon coated $\mathrm{Au} / \mathrm{TiO}_{2}$ mesoporous microspheres: a novel selective photocatalyst. Sci China Mater, 2017, 60: 438-448

44 Xing R, Liu K, Jiao T, et al. An injectable self-assembling collagengold hybrid hydrogel for combinatorial antitumor photothermal/ photodynamic therapy. Adv Mater, 2016, 28: 3669-3676

45 Mathiyazhakan M, Upputuri PK, Sivasubramanian K, et al. In situ synthesis of gold nanostars within liposomes for controlled drug release and photoacoustic imaging. Sci China Mater, 2017, 59: 892900

46 Guo W, Jiao J, Tian K, et al. Controllable synthesis of core-satellite $\mathrm{Fe}_{3} \mathrm{O}_{4} @$ polypyrrole/Pd nanoarchitectures with aggregation-free Pd nanocrystals confined into polypyrrole satellites as magnetically recoverable and highly efficient heterogeneous catalysts. RSC Adv, 2015, 5: 102210-102218

47 Guo R, Jiao T, Xing R, et al. Hierarchical AuNPs-loaded $\mathrm{Fe}_{3} \mathrm{O}_{4} /$ polymers nanocomposites constructed by electrospinning with enhanced and magnetically recyclable catalytic capacities. Nanomaterials, 2017, 7: 317

48 Zhao X, Jiao T, Ma X, et al. Facile fabrication of hierarchical diamond-based AuNPs-modified nanocomposites via layer-bylayer assembly with enhanced catalytic capacities. J Taiwan Institute Chem Engineers, 2017, 80: 614-623

49 Hervés P, Pérez-Lorenzo M, Liz-Marzán LM, et al. Catalysis by metallic nanoparticles in aqueous solution: model reactions. Chem Soc Rev, 2012, 41: 5577-5587

50 Zhao MQ, Ren CE, Ling Z, et al. Flexible MXene/carbon nanotube composite paper with high volumetric capacitance. Adv Mater, 2015, 27: 339-345

51 Li K, Jiao T, Xing R, et al. Fabrication of hierarchical MXene-based AuNPs-containing core-shell nanocomposites for high efficient catalysts. Green Energ Environ, 2018, DOI: 10.1016/j. gee.2017.11.004

52 Xing R, Wang W, Jiao T, et al. Bioinspired polydopamine sheathed nanofibers containing carboxylate graphene oxide nanosheet for high-efficient dyes scavenger. ACS Sustain Chem Eng, 2017, 5: 4948-4956

53 Zhang R, Xing R, Jiao T, et al. Carrier-free, chemophotodynamic dual nanodrugs via self-assembly for synergistic antitumor therapy. ACS Appl Mater Interfaces, 2016, 8: 13262-13269
54 Huo S, Duan P, Jiao T, et al. Self-assembled luminescent quantum dots to generate full-color and white circularly polarized light. Angew Chem Int Ed, 2017, 56: 12174-12178

55 Zhao X, Ma K, Jiao T, et al. Fabrication of hierarchical layer-bylayer assembled diamond-based core-shell nanocomposites as highly efficient dye absorbents for wastewater treatment. Sci Rep, 2017, 7: 44076

56 Liu Y, Ma K, Jiao T, et al. Water-insoluble photosensitizer nanocolloids stabilized by supramolecular interfacial assembly towards photodynamic therapy. Sci Rep, 2017, 7: 42978

57 Guo R, Jiao T, Li R, et al. Sandwiched $\mathrm{Fe}_{3} \mathrm{O}_{4} /$ carboxylate graphene oxide nanostructures constructed by layer-by-layer assembly for highly efficient and magnetically recyclable dye removal. ACS Sustain Chem Eng, 2018, 6: 1279-1288

58 Zhou J, Liu Y, Jiao T, et al. Preparation and enhanced structural integrity of electrospun poly( $\varepsilon$-caprolactone)-based fibers by freezing amorphous chains through thiol-ene click reaction. Colloids Surfs A-Physicochem Eng Aspects, 2018, 538: 7-13

59 Xing R, Jiao T, Liu Y, et al. Co-assembly of graphene oxide and albumin/photosensitizer nanohybrids towards enhanced photodynamic therapy. Polymers, 2016, 8: 181

60 Guo H, Jiao T, Zhang Q, et al. Preparation of graphene oxidebased hydrogels as efficient dye adsorbents for wastewater treatment. Nanoscale Res Lett, 2015, 10: 272

61 Luo X, Ma K, Jiao T, et al. Graphene oxide-polymer composite langmuir films constructed by interfacial thiol-ene photopolymerization. Nanoscale Res Lett, 2017, 12: 99

62 Chen JS, Liu H, Qiao SZ, et al. Carbon-supported ultra-thin anatase $\mathrm{TiO}_{2}$ nanosheets for fast reversible lithium storage. J Mater Chem, 2011, 21: 5687-5692

63 Hou C, Jiao T, Xing R, et al. Preparation of $\mathrm{TiO}_{2}$ nanoparticles modified electrospun nanocomposite membranes toward efficient dye degradation for wastewater treatment. J Taiwan Institute Chem Engineers, 2017, 78: 118-126

$64 \mathrm{Hu} \mathrm{H}$, Xin $\mathrm{JH}, \mathrm{Hu} \mathrm{H}$, et al. Synthesis and stabilization of metal nanocatalysts for reduction reactions - a review. J Mater Chem A, 2015, 3: 11157-11182

65 Pradhan N, Pal A, Pal T. Catalytic reduction of aromatic nitro compounds by coinage metal nanoparticles. Langmuir, 2001, 17: 1800-1802

66 Ma N, Liu X, Yang Z, et al. Carrageenan asissted synthesis of palladium nanoflowers and their electrocatalytic activity toward ethanol. ACS Sustain Chem Eng, 2017, acssuschemeng.7b03425

67 Wang $\mathrm{Q}, \mathrm{Bi} \mathrm{L}, \mathrm{Ye} \mathrm{W}$, et al. Regulation of structure and ionic intercalation of colloidal nanocrystal assembly. Colloids Surfs APhysicochem Eng Aspects, 2018, 538: 229-237

68 Xue J, Han G, Ye W, et al. Structural regulation of $\mathrm{PdCu}_{2}$ nanoparticles and their electrocatalytic performance for ethanol oxidation. ACS Appl Mater Interfaces, 2016, 8: 34497-34505

69 Sang Y, Cui Y, Li Z, et al. Electrochemical reaction of nitrobenzene and its derivatives on glassy carbon electrode modified with $\mathrm{MnFe}_{2} \mathrm{O}_{4}$ colloid nanocrystal assemblies. Sensors Actuators BChem, 2016, 234: 46-52

70 Guo $\mathrm{P}$, Cui L, Wang $\mathrm{Y}$, et al. Facile synthesis of $\mathrm{ZnFe}_{2} \mathrm{O}_{4}$ nanoparticles with tunable magnetic and sensing properties. Langmuir, 2013, 29: 8997-9003

71 Guo P, Zhang G, Yu J, et al. Controlled synthesis, magnetic and photocatalytic properties of hollow spheres and colloidal nanocrystal clusters of manganese ferrite. Colloids Surfs A-Physicochem Eng Aspects, 2012, 395: 168-174 
72 Tan L, Chen D, Liu H, et al. A silica nanorattle with a mesoporous shell: an ideal nanoreactor for the preparation of tunable gold cores. Adv Mater, 2010, 22: 4885-4889

Acknowledgements This work was supported by the National Natural Science Foundation of China (21473153 and 51771162), Support Program for the Top Young Talents of Hebei Province, China Postdoctoral Science Foundation (2015M580214), and the Scientific and Technological Research and Development Program of Qinhuangdao City (201701B004).
Author contributions Li K, Jiao T, and Peng Q performed and designed the project and experiments. Xing R, Zou G, and Zhou J synthesized the nanomaterials. Zhang L, Zhou J and Peng Q characterized the materials and discussed the results of the experiments. All the authors commented on the final paper.

Conflict of interest The authors declare no conflicts of interest.

Supplementary information Supporting information is available in the online version of the paper.

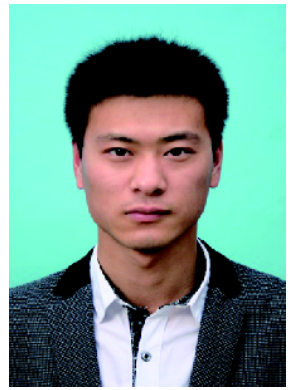

Kaikai Li is a postgraduate student in Prof. Jiao's Group and will receive his Master degree from the School of Environmental and Chemical Engineering at Yanshan University in 2018. His current research interest is MXene-based composite materials for environmental applications.
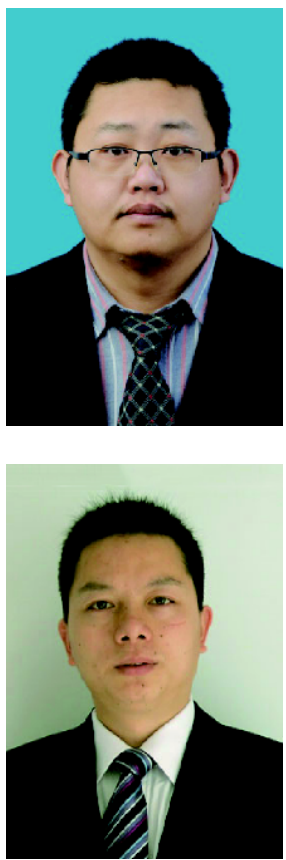

Tifeng Jiao received his $\mathrm{PhD}$ in physical chemistry from the Institute of Chemistry, Chinese Academy of Sciences (CAS). He was a postdoctoral fellow of CNRS (Centre National de la Recherche Scientifique) with A.P. Girard-Egrot (Université Claude Bernard Lyon 1, France). Currently, he is a full professor and vice director at the School of Environmental and Chemical Engineering, Yanshan University. His current research interests include synthesis of new self-assembled nanostructured materials and nanocomposites, and their related properties.
Qiuming Peng received his BSc at Xiangtan University of Technology and his $\mathrm{PhD}$ in inorganic chemistry from Changchun Institute Applied Chemistry, Chinese Academy of Sciences (CAS). He was an Alexander von Humboldt fellow with Prof. Karl Ulrich Kainer (GKSS, Germany). In 2011, he was appointed as a professor at Yanshan University. His current research interests include high-pressure metallic based materials and their related mechanical-chemical properties.

\section{自还原反应制备可调层状MXene@AuNPs复合材料以提高催化性能}

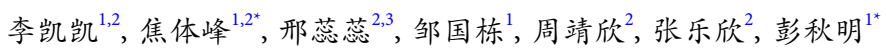

摘要 MXene是一种不同于石墨烯的新型层状二维过渡金属碳化物材料, 近年来表现出有趣的化学、物理性能并被广泛应用. 本文报道 了结构新颖且尺寸可调的自组装层状MXene@AuNPs纳米复合材料的制备方法. 在室温下MXene@AuNPs纳米复合材料可通过MXene材 料在 $\mathrm{HAuCl}_{4}$ 溶液中的自还原反应制备. 控制自还原反应的时间可精确地调节 Au粒子的颗粒尺寸. 而金纳米颗粒的粒径对 MXene@AuNPs 复合材料的催化性能具有很大的影响. 适当自还原时间下得到的MXene@AuNPs复合材料表现出对模型硝基化合物(如4-NP和2-NA)催化 反应很高的催化性能和良好的循环稳定性. 本研究工作为基于MXene自组装复合材料的制备与调控提供了新方法. 